\title{
Early Experiences with Nivolumab in Patients with Metastatic Renal Cell Carcinoma and Failed Target Therapy
}

\author{
Jong Hoon Lee ${ }^{1}$, Minyong Kang', Jae Hoon Chung', Hyun Hwan Sung', \\ Hwang Gyun Jeon ${ }^{1}$, Byung Chang Jeong ${ }^{1}$, Seong Soo Jeon ${ }^{1}$, Hyun Moo Lee', \\ Se Hoon Park', Binnari Kim ${ }^{3}$, Ghee Young Kwon ${ }^{3}$, Seong II Seo ${ }^{1}$ \\ Departments of ${ }^{1}$ Urology, ${ }^{2}$ Hemato-Oncology, ${ }^{3}$ Pathology and Translational Genomics, Samsung Medical \\ Center, Sungkyunkwan University School of Medicine, Seoul, Korea
}

\begin{abstract}
Purpose: The efficacy of nivolumab in metastatic renal cell carcinoma (mRCC) has been proven. However, the nivolumab experience in Korean patients with $\mathrm{mRCC}$ is still poorly reported. We report initial experiences with the efficacy and safety of nivolumab in patients with mRCC.

Materials and Methods: We retrospectively reviewed records for 25 patients with mRCC who had failed targeted therapy and were treated by nivolumab (2 $\mathrm{mg} / \mathrm{kg}$, every 2 weeks) at a single institution. The primary endpoint was objective response rate (ORR), and secondary endpoints were progression-free survival (PFS), safety profiles, and ORR in a programmed cell death receptor ligand 1 (PD-L1) expression subgroup.

Results: The median age was 60 years and 16 patients $(64 \%)$ were male. Objective responses were achieved in 8 patients (32.0\%) (complete response, 1; partial response, 7). Median PFS was 3.0 months (95\% confidence interval, 1.46-4.53). Treatment-related adverse events (AEs) of any grade were observed in 19 patients $(76.0 \%)$ with $6(24.0 \%)$ experiencing grade 3 to 4 treatment-related AEs. In subgroups by PD-L1 expression levels classified as $1 \%$ or greater and less than $1 \%$, ORR was $50 \%$ and $0 \%$, respectively.

Conclusions: This study showed the efficacy and safety of initial experiences with nivolumab in Korean patients with $\mathrm{mRCC}$ who had failed targeted therapy. Our results were comparable to recent clinical trials on nivolumab in mRCC. (Korean J Urol Oncol 2019;17:96-102)
\end{abstract}

Key Words: Metastasis - Monoclonal antibody $\cdot$ Programmed cell death 1 ligand $1 \cdot$ Renal cell carcinoma $\cdot$ Treatment outcome

Received June 4, 2019, Revised June 24, 2019,

Accepted July 9, 2019

Corresponding Author: Seong Il Seo

Department of Urology, Samsung Medical Center, Sungkyunkwan University School of Medicine, 81 Irwon-ro, Gangnam-gu, Seoul 06351, Korea

E-mail: seongil.seo@samsung.com

Tel: +82-2-3410-3556, Fax: +82-2-3410-6992

ORCID code: https://orcid.org/0000-0002-9792-7798

- This study was funded by a research grant from the National Research Foundation (NRF) of Korea, funded by the Ministry of Science and ICT (2018M3A9H1078336). This research was also supported by a grant of the Korea Health Technology R\&D Project through the Korea Health Industry Development Institute (KHIDI), funded by the Ministry of Health \& Welfare, Republic of Korea (HI17C0025).

\section{INTRODUCTION}

Every year, 338,000 renal cell carcinoma (RCC) patients are diagnosed worldwide ${ }^{1}$ and about $30 \%$ of patients have metastatic disease at the time of diagnosis. ${ }^{2}$ Many target therapies have been approved to treat advanced or metastatic RCC (mRCC) including vascular endothelial growth factor (VEGF) pathway inhibitors and mammalian target rapamycin (mTOR) inhibitors. VEGF pathway and mTOR inhibitors have been the main treatment agents for $\mathrm{mRCC}$ in the last decade. However,

(i) (3) This is an Open Access article distributed under the terms of the Creative Commons Attribution Non-Commercial License (http://creativecommons.org/licenses/by-nc/4.0/) which permits unrestricted non-commercial use, distribution, and reproduction in any medium, provided the original work is properly cited. 2019 (C) Copyright The Korean Urological Oncology Society and The Korean Prostate Society. All Rights Reserved. 
limitations of these drugs are in adverse events (AEs) and durable response. ${ }^{3}$ Immuno-oncology drugs such as anti-programmed death 1 (PD1) and anti-CTLA4 have received attention for $\mathrm{mRCC}$ due to their long durable response and relatively fewer AEs.

Nivolumab is a fully human IgG4 antibody. It is a PD-1 immune checkpoint inhibitor that selectively blocks the interaction between PD-1, which is expressed on activated $\mathrm{T}$ cells, and programmed cell death receptor ligand 1 (PD-L1) or 2 (PD-L2), which are expressed on immune cells and tumor cells. ${ }^{4-6}$ This is the first approved agent for use in treatment-refractory clear cell RCC. $^{7}$ Motzer et al. ${ }^{7}$ reported that nivolumab is effective and safe as a second-line treatment for advanced RCC. In a phase III trial, overall survival (OS) was longer and fewer grade 3 or 4 AEs occurred for nivolumab versus everolimus.

In Japanese patients, the efficacy and safety of nivolumab for mRCC was different from a previous global study. ${ }^{8}$ Therefore, the efficacy and safety of immune checkpoint inhibitors for mRCC is expected to also be different in Korean populations compared to global populations. However, the experience for nivolumab in Korean patients with $\mathrm{mRCC}$ is still poorly reported. We report initial experiences on the efficacy and safety of nivolumab in Korean patients with mRCC previously treated with targeted therapy.

\section{MATERIALS AND METHODS}

\section{Patients and Study Design}

We retrospectively reviewed clinical data for 25 patients with mRCC who had failed targeted therapy from March 2017 through September 2018. All included patients were treated with nivolumab as second-line treatment for mRCC. Nivolumab was administered at $2 \mathrm{mg}$ per $\mathrm{kg}$ body weight as a 60 -minute intravenous infusion every 2 weeks. This study protocol was approved by the Institutional Review Board of Samsung Medical Center (IRB No. 2019-04-160). Informed consent was exempted by the board.

Assessed baseline characteristics were age, sex, body mass index, Heng risk factor, Eastern Cooperative Oncology Group (ECOG) performance status, metastasis status, and treatment history. The primary endpoint was objective response rate (ORR) defined as the number of patients with a complete response (CR), partial response (PR), stable disease (SD), or progression of disease (PD). The best objective response was as- sessed by a urologist and uro-radiologist. Secondary endpoints included OS, progression-free survival (PFS), and safety profiles. The efficacy of nivolumab according to PD-L1 expression level was also assessed.

Disease assessments were performed using computed tomography or magnetic resonance imaging at baseline and every 6 to 8 weeks until disease progression or treatment discontinuation. Imaging data were evaluated for tumor response according to Response evaluation criteria in solid tumors version 1.1. ${ }^{9}$ OS was defined as the time from first dose to date of death. PFS was defined as time from nivolumab treatment to first documented RECIST-defined tumor progression or death from any cause.

Immunochemistry of tumor cells was performed using Dako PD-L1 immunostaining kits (Agilent, Santa Clara, CA, USA). Expression levels were classified according to presence or absence of quantifiable PD-L1 and were classified as more than $1 \%$ and less than $1 \%$, as determined by a pathologist.

Patients were allowed to continue nivolumab after initial disease progression if clinical benefit was assessed and nivolumab had an acceptable side-effect profile. Safety assessments were conducted at each clinic visit. After treatment discontinuation, patient's last outpatient visit was confirmed for assessment of survival. AEs were graded according to the National Cancer Institute Common Terminology Criteria for Adverse Events, version 4.0.

\section{Statistical Analysis}

PFS and OS were estimated using Kaplan-Meier methodology. Statistical analysis was conducted using IBM SPSS Statistics ver. 23.0 (IBM Co., Armonk, NY, USA) with a p-value $<0.05$ considered statistically significant.

\section{RESULTS}

\section{Patient Characteristics}

Baseline characteristics of the 25 patients are in Table 1 . Median age was 60 years and most were male (64\%). Of the patients, $4(16 \%)$ were classified by Heng criteria as having favorable risk, $16(64 \%)$ as having intermediate risk and $5(20 \%)$ as having poor risk. Most patients had $0(20 \%, \mathrm{n}=5)$ or $1(76 \%$, $\mathrm{n}=19)$ ECOG performance status. Metastatic types were synchronous in 16 patients (64\%) and metachronous in 9 (36\%) with 20 patients $(80 \%)$ having $\geq 2$ metastasis sites. Metastasis sites were lung $(88 \%, \mathrm{n}=22)$, lymph node $(68 \%, \mathrm{n}=17)$, bone 
Table 1. Baseline clinical characteristics of 25 patients with failed targeted therapy who were treated with nivolumab for metastatic renal cell carcinoma

\begin{tabular}{|c|c|}
\hline Characteristic & Value \\
\hline Age (yr), median (IQR) & $60(54-66)$ \\
\hline \multicolumn{2}{|l|}{ Sex } \\
\hline Male & $16(64)$ \\
\hline Female & $9(36)$ \\
\hline $\begin{array}{l}\text { Body mass index }\left(\mathrm{kg} / \mathrm{m}^{2}\right) \text {, median } \\
(\mathrm{IQR})\end{array}$ & $22.97(20.29-25.48)$ \\
\hline \multicolumn{2}{|l|}{ Heng risk group } \\
\hline Favorable & $4(16)$ \\
\hline Intermediate & $16(64)$ \\
\hline Poor & $5(20)$ \\
\hline \multicolumn{2}{|l|}{ ECOG performance status } \\
\hline $0-1$ & $24(96)$ \\
\hline 2 & $1(4)$ \\
\hline \multicolumn{2}{|l|}{ Histopathology } \\
\hline Clear cell RCC & $22(88)$ \\
\hline Nonclear cell RCC & $3(12)$ \\
\hline \multicolumn{2}{|l|}{ Type of metastasis } \\
\hline Synchronous & $16(64)$ \\
\hline Metachronous & $9(36)$ \\
\hline \multicolumn{2}{|l|}{ No. of metastasis } \\
\hline Single & $5(20)$ \\
\hline Multiple $(\geq 2)$ & $20(80)$ \\
\hline \multicolumn{2}{|l|}{ Site of metastasis } \\
\hline Lung & $22(88)$ \\
\hline Lymph node & $17(68)$ \\
\hline Bone & $12(48)$ \\
\hline Liver & $4(16)$ \\
\hline Adrenal & $3(12)$ \\
\hline Other soft tissue & $3(12)$ \\
\hline Prior nephrectomy & $23(92)$ \\
\hline Prior metastasectomy & $7(28)$ \\
\hline Prior radiotherapy & $9(36)$ \\
\hline \multicolumn{2}{|l|}{ No. of prior systemic regimens } \\
\hline 1 & $7(28)$ \\
\hline 2 & $9(36)$ \\
\hline$\geq 3$ & $9(26)$ \\
\hline \multicolumn{2}{|l|}{ Type of previous systemic regimens } \\
\hline Sunitinib & $11(44)$ \\
\hline Pazopanib & $14(56)$ \\
\hline Sorafenib & $7(28)$ \\
\hline Axitinib & $5(20)$ \\
\hline Everolimus & $15(60)$ \\
\hline Interleukin-2 & $2(8)$ \\
\hline $\begin{array}{l}\text { Patients with quantifiable PD-L1 } \\
\text { expression }\end{array}$ & $5(25)$ \\
\hline \multicolumn{2}{|l|}{ PD-L1 expression level } \\
\hline$\geq 1 \%$ & $4(80)$ \\
\hline$<1 \%$ & $1(20)$ \\
\hline
\end{tabular}

Table 1. Continued

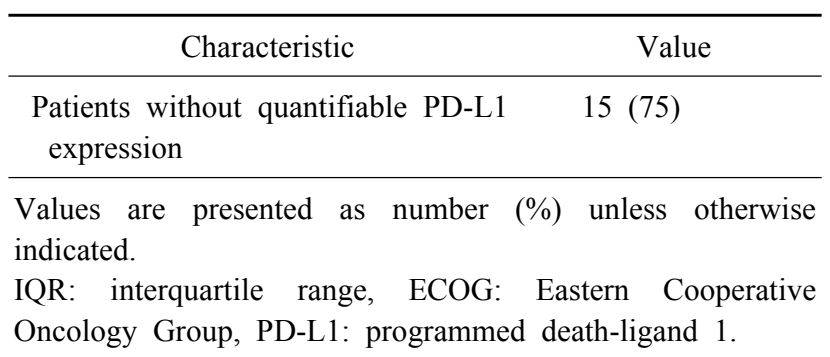

Table 2. Efficacy of nivolumab treatment in patients with metastatic renal cell carcinoma previously treated with targeted therapy

\begin{tabular}{ll}
\hline \multicolumn{1}{c}{ Outcomes } & Value \\
\hline Objective response rate* & $8(32)$ \\
Best objective response* & $1(4)$ \\
Complete response & $7(28)$ \\
Partial response & $8(32)$ \\
Stable disease & $8(32)$ \\
Progressive disease & \\
Progression-free survival (mo) & $3.0(1.4-4.6)$ \\
Median $(95 \% \mathrm{CI})$ & \\
Overall survival & Not reached \\
Median $(95 \% \mathrm{CI})$ &
\end{tabular}

Values are presented as number (\%) unless otherwise indicated.

CI: confidence interval.

*One patient has not undergone response evaluation yet among a total of 25 patients.

(48\%, $n=12)$, liver $(16 \%, n=4)$, adrenal $(12 \%, n=3)$, and other soft tissue $(12 \%, n=3)$. Of the patients, $23(92 \%)$ had prior nephrectomy and 7 (28\%) had metastatectomy with 18 (62\%) receiving at least 2 prior systemic treatments for RCC.

\section{Efficacy}

Objective responses were observed in $32 \%$ of patients ( 8 of 25) (Table 2). One patient has not undergone response evaluation yet among a total of 25 patients. Median time to response was 2.4 months (interquartile range, 1.3-5.2) among the 8 patients with a response: 1 (4\%) showed CR, 7 (28\%) showed PR, and $8(32 \%)$ showed stable SD. Among patients with a treatment response, only $1(12.5 \%)$ had a durable response (18 months). Patients 1 and 5 had a response at the time of analysis, and subsequent follow-up was not reflected in the results (Fig. 1). Median PFS was 3 months (95\% confidence interval, 1.38-4.61). Median OS was not reached (Fig. 2). 


\section{PD-L1 Expression}

Among 25 patients, 20 tissues were available to be analyzed. Quantifiable PD-L1 expression was observed in 5 patients

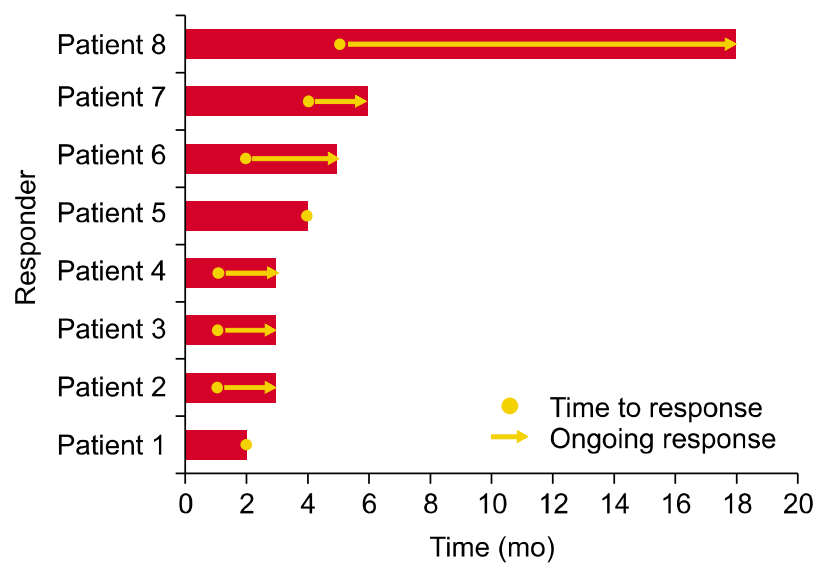

Fig. 1. Duration of response in patients who showed objective response (CR plus PR). CR: complete response, PR: partial response.

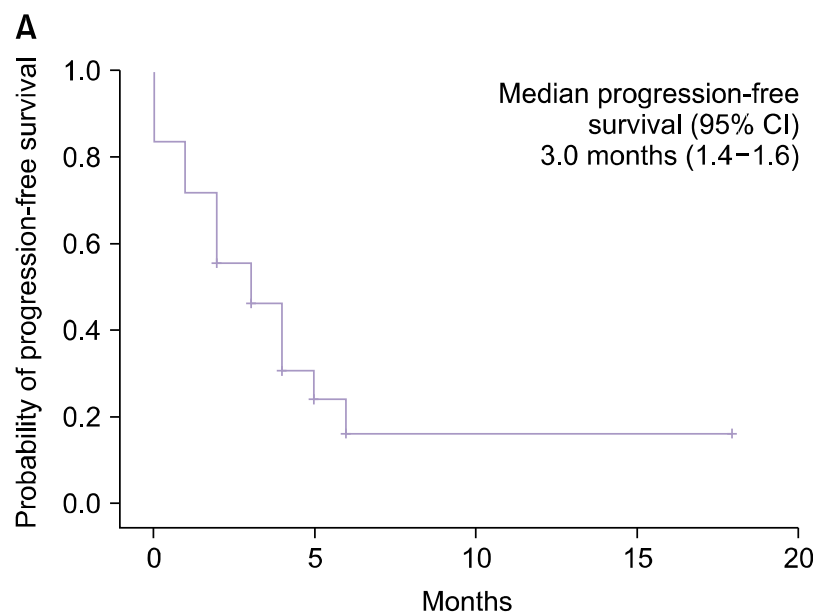

No. of patients at risk

25

9

9

9
(25\%): 1 of 5 (20\%) had less than 1\% PD-L1 expression and $4(80 \%)$ had more than 1\% PD-L1 expression. Quantifiable PD-L1 expression levels in 5 patients and corresponding ORR are in Table 3. Patients with less than 1\% PD-L1 expression showed no objective response. Two patients with PD-L1 expression $\geq 1 \%$ showed objective response. Representative im-

Table 3. Antitumor activity by PD-L1 expression level

\begin{tabular}{lcc}
\hline Variable & PD-L1 $<1 \%$ PD-L1 $\geq 1 \%$ \\
\hline No. of patients & 1 & 4 \\
Objective response rate & 0 & 2 \\
Best objective response & & \\
Complete response & 0 & 0 \\
Partial response & 0 & 2 \\
Stable disease & 0 & 1 \\
Progressive disease & 1 & 1 \\
\hline
\end{tabular}

Quantifiable PD-L1 expression was observed in 5 patients (25\%).

PD-L1: programmed death-ligand 1.

B

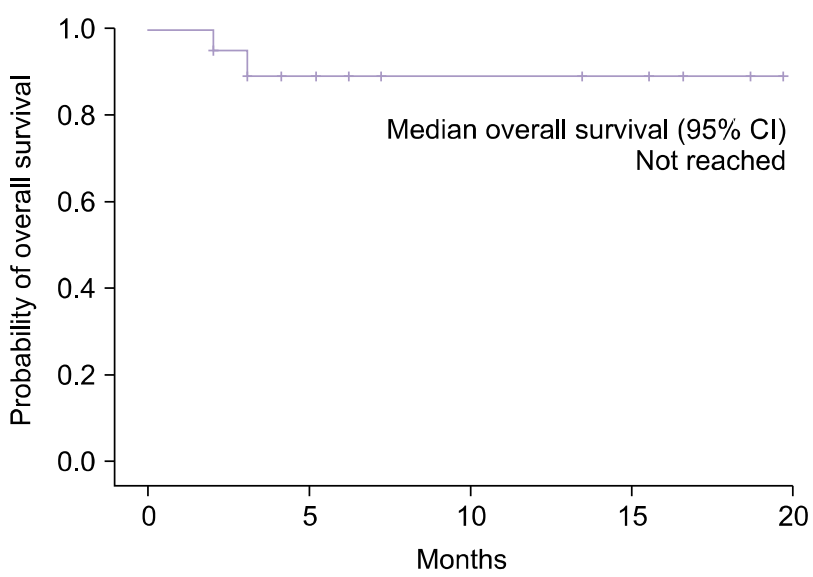

No. of patients at risk

25

23

23

23

23

Fig. 2. Kaplan-Meier curves for progression-free survival (A) and overall survival (B). CI: confidence interval.
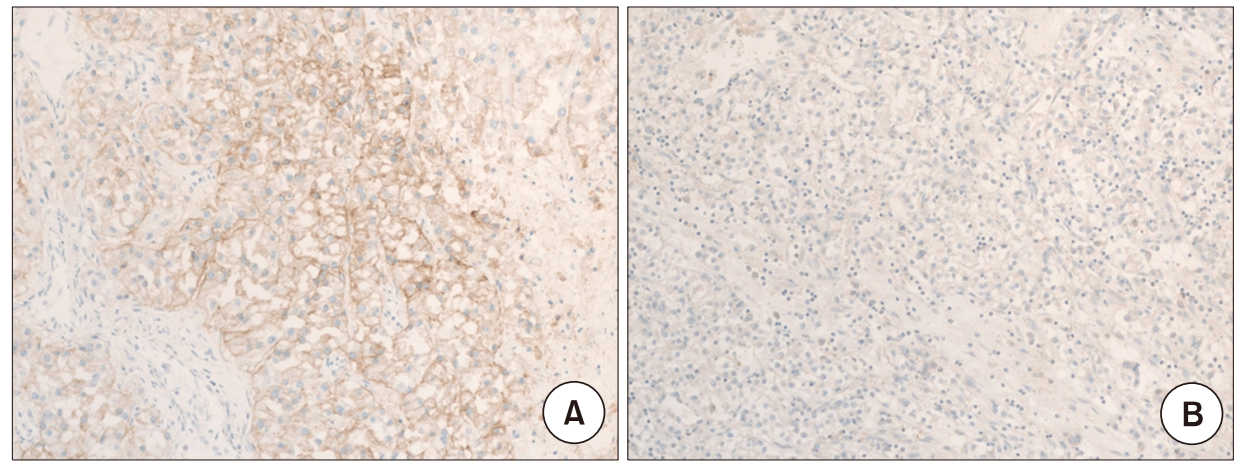

Fig. 3. Representative PD-L1 immunohistochemistry staining in renal cell carcinoma at $\times 200$ magnification. (A) PD-L1 positive case. Most of the tumor cells showed complete or partial membranous staining. (B) PD-L1 negative case of renal cell carcinoma. PD-L1: programmed death-ligand 1 . 
Table 4. Treatment-related adverse events (AEs)

\begin{tabular}{lrc}
\hline \multicolumn{1}{c}{ Adverse event } & Any grade & Grade 3 or 4 \\
\hline Any treatment-related AE & $19(76)$ & $6(24)$ \\
Nausea & $9(36)$ & $1(4)$ \\
Decreased appetite & $7(28)$ & $1(4)$ \\
Fatigue & $7(28)$ & $1(4)$ \\
Pruritis & $5(20)$ & $2(8)$ \\
Cough & $4(16)$ & $1(4)$ \\
Pneumonitis & $1(4)$ & $0(0)$ \\
Hyperthyroidism & $1(4)$ & $0(0)$ \\
Others* & $6(24)$ & $0(0)$ \\
\hline
\end{tabular}

Values are presented as number (\%).

*Other adverse events: only one case of mucositis, tinnitus, chest discomfort, sweating, melena, and insomnia were reported among 25 patients, respectively.

ages of positive and negative PD-L1 expression are in Fig. 3.

\section{Safety}

Treatment-related AEs for all patients are in Table 4. Treatment-related AEs of any grade occurred in 19 of 25 patients $(76 \%)$. The most common treatment-related AEs were nausea (9 patients, 36\%), decreased appetite (7 patients, $28 \%$ ), fatigue (7 patients, 28\%), and pruritis (5 patients, 20\%). Grade 3 or 4 treatment-related AEs occurred in 6 of the 25 patients (24\%). The most common grade 3 or 4 event was pruritis (2 patients, $8 \%$ ). No patients discontinued treatment due to treatment-related AEs and no deaths from drug toxic effects were reported.

\section{DISCUSSION}

To our knowledge, this study is the first reported the effectiveness and safety of nivolumab for Korean patients with mRCC who had failed targeted therapy. We also found that the effect of an anti-PD1 inhibitor was dependent on PD-L1 expression level.

RCC is histologically known as an immune-related disease. ${ }^{10}$ Interferon- $\alpha$ (INF- $\alpha)$ and interleukin-2 (IL-2) have mainly been used to treat mRCC. ${ }^{11}$ However, about $5 \%$ of patients showed a durable response to high-dose IL-2 but with significant AEs. ${ }^{12,13}$ The treatment of $\mathrm{mRCC}$ has seen major changes since 2005. Several tyrosine kinase inhibitors (TKIs) targeting the VEGF pathway and mTOR inhibitors have been approved for managing mRCC. Pazopanib, sunitinib, temsirolimus, and bev- acizumab (in combination with INF- $\alpha$ ) are approved as first-line treatment and everolimus, sorafenib, axitinib, and cabozantinib as second-line treatment. ${ }^{14-18}$ Despite significant progress in treatment outcomes with these agents, the median OS for patients with mRCC is only 22-29 months. ${ }^{18,19}$ Remaining target therapies do not show significant OS benefits other than temsirolimus treatment. ${ }^{20}$ Therefore, new treatments for $\mathrm{mRCC}$ are needed.

Immunotherapy for solid tumors is a possible less-toxic form of immune checkpoint inhibitors. These treatments inhibit innate immune inhibition by blocking PD-1 among T-cell receptors, as well as targeting PD-L1 and PD-L2 expressed in cancer cells. Expression of PD-1, PD-L1 and PD-L2 in cancer cells induces tumor resistance to the immune response and tumor growth. PD-1, PD-L1, and PD-L2 inhibitors that block this resistance mechanism can improve the host immune response to tumors. ${ }^{21,22}$

In a large-scale, long-term, phase III trial, the immune checkpoint inhibitor nivolumab showed superior OS and higher ORR than everolimus for mRCC patients who had previous treatment such as TKIs. ${ }^{7}$ In that trial, ORR was about $25 \%$ in the nivolumab group. ${ }^{7}$ Our study had an ORR that was comparable to the previous study.

PFS in our study was shorter than in previous studies. ${ }^{7,8}$ Unlike previous studies that evaluated the efficacy of nivolu$\mathrm{mab}$ as a second-line treatment after failure of first-line treatment, a majority of patients in our study received more than third-line nivolumab treatment. This difference may be the cause of differing results from previous studies.

In earlier studies, any grade AEs occurred in $79 \%$ of patients and grades 3-4 AEs in 19\%. ${ }^{7}$ A similar incidence rate of AEs was obtained in a Japanese subgroup ${ }^{8}$ and in our study. This similarity suggests that the incidence of AEs of nivolumab is consistent regardless of race.

When efficacy according to PD-L1 expression was confirmed by immunohistochemical staining, OS was 21.8 in group with more than $1 \%$ expression and 27.4 months in a group with less than $1 \%$ expression in a previous study. ${ }^{7}$ This result, although not evaluated for statistical significance, was contrary to expectations. In addition, no comparative analysis of ORR according PD-L1 expression level was performed. In contrast, in our study, ORR was higher in patients in the group with more than $1 \%$ expression. Although there was no statistical significance, this result suggested that the expression levels of 
PD-L1 and nivolumab efficacy may be related. However, the number of patients who underwent immunohistochemical staining was small, so validation of PD-L1 expression is required in a larger number of patients.

Our study had several limitations. It was retrospective and therefore had unavoidable bias such as bias in selection. A lack of clinical data and possible misclassification of registered patients may have influenced our results. Our study included a small number of patients and we used only a single tertiary center database. The median follow-up of our study was short, at 5 months. Because of this length, OS was not reached. The heterogeneity of previous systemic therapies may have produced more inferior results than expected.

\section{CONCLUSIONS}

In conclusion, we reported the initial experiences with anti-PD1 (nivolumab) treatment in Korean patients with mRCC who had failed targeted therapy. Our efficacy and safety data were comparable to data from recent clinical trials on nivolumab in mRCC. Our data provide valuable information on immuno-oncology drugs for treating $\mathrm{mRCC}$ that will be useful for real-world clinical practice.

\section{CONFLICT OF INTEREST}

The authors claim no conflicts of interest.

\section{REFERENCES}

1. Ferlay J, Soerjomataram I, Dikshit R, Eser S, Mathers C, Rebelo $\mathrm{M}$, et al. Cancer incidence and mortality worldwide: sources, methods and major patterns in GLOBOCAN 2012. Int J Cancer 2015;136:E359-86.

2. Fisher R, Gore M, Larkin J. Current and future systemic treatments for renal cell carcinoma. Semin Cancer Biol 2013;23: $38-45$.

3. Escudier B, Porta C, Schmidinger M, Algaba F, Patard JJ, Khoo V, et al. Renal cell carcinoma: ESMO Clinical Practice Guidelines for diagnosis, treatment and follow-up. Ann Oncol 2014;25 Suppl 3:iii49-56.

4. Hamanishi J, Mandai M, Iwasaki M, Okazaki T, Tanaka Y, Yamaguchi K, et al. Programmed cell death 1 ligand 1 and tumor-infiltrating CD8+ $\mathrm{T}$ lymphocytes are prognostic factors of human ovarian cancer. Proc Natl Acad Sci U S A 2007; 104:3360-5.
5. Hamid O, Carvajal RD. Anti-programmed death-1 and anti-programmed death-ligand 1 antibodies in cancer therapy. Expert Opin Biol Ther 2013;13:847-61.

6. Nurieva RI, Liu X, Dong C. Molecular mechanisms of T-cell tolerance. Immunol Rev 2011;241:133-44.

7. Motzer RJ, Escudier B, McDermott DF, George S, Hammers HJ, Srinivas S, et al. Nivolumab versus Everolimus in Advanced Renal-Cell Carcinoma. N Engl J Med 2015;373: 1803-13.

8. Tomita Y, Fukasawa S, Shinohara N, Kitamura H, Oya M, Eto $\mathrm{M}$, et al. Nivolumab versus everolimus in advanced renal cell carcinoma: Japanese subgroup analysis from the CheckMate 025 study. Jpn J Clin Oncol 2017;47:639-46.

9. Eisenhauer EA, Therasse P, Bogaerts J, Schwartz LH, Sargent $\mathrm{D}$, Ford R, et al. New response evaluation criteria in solid tumours: revised RECIST guideline (version 1.1). Eur $\mathrm{J}$ Cancer 2009;45:228-47.

10. Itsumi M, Tatsugami K. Immunotherapy for renal cell carcinoma. Clin Dev Immunol 2010;2010:284581.

11. Molina AM, Motzer RJ, Heng DY. Systemic treatment options for untreated patients with metastatic clear cell renal cancer. Semin Oncol 2013;40:436-43.

12. McDermott DF, Atkins MB. Immunotherapy of metastatic renal cell carcinoma. Cancer J 2008;14:320-4.

13. Klapper JA, Downey SG, Smith FO, Yang JC, Hughes MS, Kammula US, et al. High-dose interleukin-2 for the treatment of metastatic renal cell carcinoma: a retrospective analysis of response and survival in patients treated in the surgery branch at the National Cancer Institute between 1986 and 2006. Cancer 2008;113:293-301.

14. Escudier B, Eisen T, Stadler WM, Szczylik C, Oudard S, Siebels M, et al. Sorafenib in advanced clear-cell renal-cell carcinoma. N Engl J Med 2007;356:125-34.

15. Escudier B, Pluzanska A, Koralewski P, Ravaud A, Bracarda $\mathrm{S}$, Szczylik C, et al. Bevacizumab plus interferon alfa-2a for treatment of metastatic renal cell carcinoma: a randomised, double-blind phase III trial. Lancet 2007;370:2103-11.

16. Motzer RJ, Hutson TE, Cella D, Reeves J, Hawkins R, Guo $\mathrm{J}$, et al. Pazopanib versus sunitinib in metastatic renal-cell carcinoma. N Engl J Med 2013;369:722-31.

17. Motzer RJ, Hutson TE, Glen H, Michaelson MD, Molina A, Eisen $\mathrm{T}$, et al. Lenvatinib, everolimus, and the combination in patients with metastatic renal cell carcinoma: a randomised, phase 2, open-label, multicentre trial. Lancet Oncol 2015;16: 1473-82.

18. Rini BI, Escudier B, Tomczak P, Kaprin A, Szczylik C, Hutson TE, et al. Comparative effectiveness of axitinib versus sorafenib in advanced renal cell carcinoma (AXIS): a randomised phase 3 trial. Lancet 2011;378:1931-9.

19. Heng DY, Xie W, Regan MM, Warren MA, Golshayan AR, Sahi C, et al. Prognostic factors for overall survival in patients with metastatic renal cell carcinoma treated with vascular en- 
dothelial growth factor-targeted agents: results from a large, multicenter study. J Clin Oncol 2009;27:5794-9.

20. Hudes G, Carducci M, Tomczak P, Dutcher J, Figlin R, Kapoor A, et al. Temsirolimus, interferon alfa, or both for advanced renal-cell carcinoma. N Engl J Med 2007;356:2271-81.

21. Brahmer JR, Tykodi SS, Chow LQ, Hwu WJ, Topalian SL, Hwu P, et al. Safety and activity of anti-PD-L1 antibody in patients with advanced cancer. N Engl J Med 2012;366:245565.

22. Topalian SL, Hodi FS, Brahmer JR, Gettinger SN, Smith DC, McDermott DF, et al. Safety, activity, and immune correlates of anti-PD-1antibody in cancer. N Engl J Med 2012;366: 2443-54. 